\title{
ON TANGENTIAL SLIT SOLUTION OF THE LOEWNER EQUATION
}

\author{
Ka-Sing Lau and Hai-Hua $\mathrm{Wu}^{*}$ \\ The Chinese University of Hong Kong, Department of Mathematics \\ Shatin, N.T., Hong Kong; kslau@math.cuhk.edu.hk \\ The Chinese University of Hong Kong, Department of Mathematics, Hong Kong \\ and Hunan University, College of Mathematics and Econometrics \\ Changsha, Hunan 410082, P. R. China; hunaniwa@163.com
}

\begin{abstract}
For a non-tangential slit $\gamma(t)$, the behavior of the driving function $\lambda(t)$ near zero in the Loewner equation is well understood; for tangential slit, the situation is less clear. In this paper, we investigate the tangential slit $\Gamma^{p}, p>0$, where $\Gamma$ is a circular arc tangent at $0 ; \Gamma^{p}$ has order $\frac{p+1}{p}$ near zero. Our main result is to give the exact expression of $\lambda(t)$, and its Hölder exponent near 0 in terms of $p$, which has a natural connection with the known results. We also extend this to a general type of tangential slits, and give an estimation of the growth of $\lambda(t)$ near 0 .
\end{abstract}

\section{Introduction}

The Loewner differential equation was introduced by Loewner in the 20's to study the Bieberbach conjecture, and was the main tool in the final solution of the conjecture by de Brange [1]. The importance of the equation emerged again in the recent study of the stochastic Loewner evolution (SLE) due to Lawler, Schramm and Werner $[9,10,11,19,8]$ and the references there, and Smirnov [20, 21, 22]. This also re-ignited the interest of the equation and its solution in the deterministic case $[2,5,6,12,13,14,15,16,18,24,25]$.

Let $\mathbf{H}$ be the upper half-plane. Suppose for any $T>0, \gamma:[0, T] \rightarrow \overline{\mathbf{H}}$ is a simple curve with $\gamma(0) \in \mathbf{R}$ and $\gamma(0, T] \subset \mathbf{H}$. For every $t \in[0, T]$, the region $H_{t}=\mathbf{H} \backslash \gamma[0, t]$ is simply connected, and by changing the parametrization of $\gamma$, there is a unique conformal map $g_{t}$ from $H_{t}$ onto $\mathbf{H}$ such that

$$
g_{t}(z)=z+\frac{2 t}{z}+O\left(\frac{1}{|z|^{2}}\right) \quad \text { as } z \rightarrow \infty .
$$

This $\gamma$ is said to be parameterized by the half-plane capacity. In this case, $g_{t}(z)$ satisfies the equation

$$
\dot{g}_{t}(z)=\frac{2}{g_{t}(z)-\lambda(t)}, \quad g_{0}(z)=z
$$

doi:10.5186/aasfm.2016.4142

2010 Mathematics Subject Classification: Primary 30C45, 30C20; Secondary 30C62, 30C30.

Key words: Loewner equation, hull, half-plane capacity, driving function, trace.

The research is supported in part by the HKRGC Grant and the NNSF of China (No. 11571099, $11371382,11301175)$, the second author is also supported by the SRFDP of Higher Education (No. 20134306110003), the Hunan Provincial NSF (No. 13JJ4042) and the SRF of Hunan Provincial Education Department (No. 14K057).

${ }^{*}$ The first and the corresponding author. 
where $\lambda(t):=\lim _{z \rightarrow \gamma(t)} g_{t}(z)$ is a continuous real-valued function. The equation (1.2) is called the (chordal) Loewner differential equation, $\lambda$ is called the driving function of the curve $\gamma$, and $\gamma$ is called the trace of $\lambda$.

On the other hand, given a continuous function $\lambda:[0, T] \rightarrow \mathbf{R}$ and $z \in \mathbf{H}$, we can solve the initial value problem (1.2). Let $T_{z}$ be the supremum of all $t$ such that the solution is well defined up to time $t$ with $g_{t}(z) \in \mathbf{H}$. Let

$$
H_{t}:=\left\{z \in \mathbf{H}: T_{z}>t\right\} .
$$

Then $g_{t}$ is the unique conformal transformation from $H_{t}$ onto $\mathbf{H}$ satisfying (1.1). Let $K_{t}:=\mathbf{H} \backslash H_{t}$. Then $\left\{K_{t}\right\}_{t \in[0, T]}$ is an increasing family of hulls in $\mathbf{H}$ (defined in Section 2), and we can say that the hulls $K_{t}$ are generated by the Loewner equation (1.2).

In general, the domains $H_{t}$ generated by a continuous driving function $\lambda$ are not necessarily slit half-planes (i.e., domains of the form $\mathbf{H} \backslash \gamma[0, t]$, for some simple continuous curve $\gamma$ in $\mathbf{H} \cup\{\gamma(0)\}$ with $\gamma(0) \in \mathbf{R})$. Recall that $\operatorname{Lip}(1 / 2)$ is the space of Hölder continuous functions with exponent 1/2. Marshall and Rohde [15] and Lind [12] proved that $H_{t}$ is a slit half-plane for all $t$ provided that $\|\lambda\|_{1 / 2}<4$; conversely, $\lambda \in \operatorname{Lip}(1 / 2)$ if $\gamma$ is a quasiarc that approaches $\mathbf{R}$ non-tangentially. For tangential slit, recently Prokhorov and Vasil'ev [16] showed that the circular arc

$$
\Gamma=\left\{i+e^{i \theta}:-\frac{\pi}{2} \leq \theta \leq \frac{\pi}{2}\right\} .
$$

is generated by a Hölder continuous driving function with exponent $1 / 3$ in the Loewner equation (1.2).

In this paper, we consider the tangent slit

$$
\Gamma^{p}:=\left\{z^{p}: z=i+e^{i \theta},-\frac{\pi}{2} \leq \theta \leq \frac{\pi}{2}\left(\theta_{p}-1\right)\right\},
$$

where $\theta_{p}=1 / p$ if $p \geq 1 ; \theta_{p}=1$ if $0<p<1$ (we take the branch such that $1^{p}=1$ ). The condition on the angle ensures that the simple smooth curve $\Gamma^{p} \backslash\{0\}$ is contained in the upper half plane, hence it is the trace of some driving function $\lambda(t)$. It is not difficult to show that for $x+i y \in \Gamma^{p}$, then $y=\frac{p}{2} x^{\frac{p+1}{p}}+o\left(x^{\frac{p+1}{p}}\right)$, i.e., $\Gamma^{p}$ is a tangent slit of order $(p+1) / p$ at 0 (Proposition 2.3). Our main theorem is

Theorem 1.1. Let $\Gamma^{p}$ be the trace generated by the Loewner equation (1.2). Then the driving function $\lambda(t)$ has the form

$$
\lambda(t)=C t^{\frac{p}{2 p+1}}+o\left(t^{\frac{p}{2 p+1}}\right), \quad \text { as } t \rightarrow 0,
$$

for some $C>0$.

We actually prove in Theorem 3.1 for a complete expression of $\lambda(t)$ in terms of a series, and the constant $C$ is given explicitly. We remark that the case in [16] is for $p=1$, and the case in [12] and [15] corresponds to $p=\infty$ heuristically. The technique of proof is to obtain an integral expression of $f_{t}=g_{t}^{-1}$ using the Christoffel-Schwarz formula; we can then reduce the integral expression to a functional equation of $\lambda(t)$, which can be handled.

In [25], Wu and Dong considered the limit of $\gamma$ within a sector, and proved that: if there exist $T>0, \alpha, \beta \in(0, \pi)$ such that $\arg \left(K_{T}\right) \subset(\alpha, \beta)$, then $\lim _{\sup _{t \rightarrow 0}}|\lambda(t)| / \sqrt{t}<$ $\infty$. For general tangent slits of order $r$ at 0 , we have the following theorem.

Theorem 1.2. Let $r>1,0<a<b$, and let $\left\{K_{t}\right\}_{t}$ be the growing hulls generated by the Loewner equation (1.2). If there exists a positive real number $T$ 
such that $K_{T}$ lies in the domain $D_{r}(a, b):=\left\{x+i y: x>0, a x^{r}<y<b x^{r}\right\}$, then

$$
\limsup _{t \rightarrow 0} \frac{|\lambda(t)|}{t^{\frac{1}{r+1}}}<\infty
$$

Clearly, the $\Gamma^{p}$ in Theorem 1.1 is the special case with $r=(p+1) / p$. We do not know the estimate of $\lim \inf _{t \rightarrow 0}|\lambda(t)| / t^{\frac{1}{r+1}}$. It is known that for $\alpha>1 / 2$, if $\lambda \in C^{\alpha}$, then $\gamma \in C^{\alpha+1 / 2}[14,15,24]$; the converse is still unknown. Our case is a supplement of this, and in particular corresponds to the case $r=1$.

\section{Preliminaries}

We call a bounded subset $A \subset \mathbf{H}$ a (compact) $\mathbf{H}$-hull if $A=\mathbf{H} \cap \bar{A}$ and $\mathbf{H} \backslash A$ is simply connected. For such hull $A$, there is a unique conformal transformation $g_{A}: \mathbf{H} \backslash A \rightarrow \mathbf{H}$ such that $\lim _{z \rightarrow \infty}\left(g_{A}(z)-z\right)=0$ [8]. The half-plane capacity is defined by

$$
\operatorname{hcap}(A):=\lim _{z \rightarrow \infty} z\left(g_{A}(z)-z\right)
$$

In other words,

$$
g_{A}(z)=z+\frac{\operatorname{hcap}(A)}{z}+O\left(\frac{1}{|z|^{2}}\right), \quad z \rightarrow \infty .
$$

Note that the half-plane capacity of the hull $K_{t}$ generated by (1.2) is equal to $2 t$. From $g_{r A}(z)=r g_{A}(z / r)$, it follows that

$$
\operatorname{hcap}(r A)=r^{2} \operatorname{hcap}(A) \text {. }
$$

The half-plane capacity can be defined in a number of equivalent ways [8], and there are various geometric interpretations for it $[7,17]$. We need the following estimations due to Lalley et al. [7] and Lind, Marshall and Rohde [13].

Theorem 2.1. [7] For a hull $A$, there exists $C>0$ such that

$$
C^{-1} \cdot \operatorname{hsiz}(A)<\operatorname{hcap}(A)<C \cdot \operatorname{hsiz}(A),
$$

where $\operatorname{hsiz}(A):=\operatorname{area}\left(\bigcup_{x+i y \in A} B(x+i y, y)\right)$, and $B(z, \eta)$ denotes the disk of radius $\eta$ about $z$.

Theorem 2.2. [13] Let $A$ be a hull, and let $g_{A}$ be the unique conformal transformation $g_{A}: \mathbf{H} \backslash A \rightarrow \mathbf{H}$ be defined as the above. Then for $\sigma=\inf \{\bar{A} \cap \mathbf{R}\}$, $\tau=\sup \{\bar{A} \cap \mathbf{R}\}$, and $I=\left[g_{A}(\sigma-), g_{A}(\tau+)\right]$, we have

$$
\operatorname{diam} A \leq \operatorname{diam} I \leq 4 \operatorname{diam} A .
$$

Suppose $\left\{K_{t}\right\}_{t \in[0, T]}$ is an increasing family of hulls generated by the Loewner equation (1.2). Let $g_{t}$ be the conformal transformation of $\mathbf{H} \backslash K_{t}$ onto $\mathbf{H}$ as in (1.1). Let $K_{t, s}$ be the hull $g_{t}\left(K_{s} \backslash K_{t}\right) \cap \mathbf{H}$ for all $t<s$. It is not hard to see that $\bigcap_{\delta>0} \overline{K_{t, t+\delta}}$ is the single point $\lambda(t)$. In particular, this implies that $\gamma(0)=\lambda(0)$ if $K_{t}=\gamma(0, t]$ for a simple curve $\gamma$.

We mention some basic properties of the chordal Loewner equation that we use. Suppose the hulls $K_{t}$ are generated by a driving function $\lambda(t)$, then we have

(i) Scaling: for $\eta>0$, the scaled hull $\eta K_{t / \eta^{2}}$ is driven by $\eta \lambda\left(t / \eta^{2}\right)$.

(ii) Translation: for $d \in \mathbf{R}$, the driving function of $K_{t}+d$ is $\lambda(t)+d$.

(iii) Reflection: the reflected hulls $R_{I}\left(K_{t}\right)$ are generated by $-\lambda(t)$, where $R_{I}$ denotes reflection in the imaginary axis. 
(iv) Concatenation: for fixed $T>0$, the mapped hulls $g_{T}\left(K_{T+t}\right)$ are driven by $\lambda(T+t)$.

The simple curve $\Gamma^{p}$ is the trace of a driving function $\lambda(t)$. The order of $\Gamma^{p}$ is given by the following simple proposition.

Proposition 2.3. For $x+i y \in \Gamma^{p}, p>0$, we have $y=\frac{p}{2} x^{\frac{p+1}{p}}+o\left(x^{\frac{p+1}{p}}\right)$ as $x \rightarrow 0$, i.e., $\Gamma^{p}$ is a tangent slit of order $(p+1) / p$.

Proof. For $p=1, \Gamma$ is the circular arc, $x^{2}+(y-1)^{2}=1$, so that $y=\frac{1}{2} x^{2}+o\left(x^{2}\right)$ as $x \rightarrow 0$. For $p \neq 1$, we have $(x+i y)^{\frac{1}{p}}=u+i v \in \Gamma$. Hence by using the binomial expansion,

$$
x+i y=\left(u+i\left(\frac{1}{2} u^{2}+o\left(u^{2}\right)\right)\right)^{p}=u^{p}\left(1+\frac{i p}{2} u+o(u)\right) .
$$

Comparing the real and imaginary parts, we have $y=\frac{p}{2} x^{\frac{p+1}{p}}+o\left(x^{\frac{p+1}{p}}\right)$ as $x \rightarrow 0$.

To close this section, we consider a functional equation which is associated with the driving function $\lambda(t)$ of $\Gamma^{p}$ (Section 3).

Lemma 2.4. Let $\varphi_{0}:[0, T] \rightarrow[0,1)$ be a continuous function such that $\varphi_{0}(0)=$ 0 , and satisfies

$$
\varphi_{0}(t)\left(1-\varphi_{0}(t)\right)^{q}=c_{1} t^{q}, \quad t \in[0, T]
$$

for some $q, c_{1}>0$. Then $\varphi_{0}(t)=\sum_{n=1}^{\infty} c_{n} t^{q n}$ with

$$
c_{n+1}=c_{1} \sum_{j=1}^{n} \sum_{\substack{i_{1}+\cdots+i_{j}=n \\ i_{1}, \cdots, i_{j} \geq 1}} \frac{q(q+1) \cdots(q+j-1)}{j !} c_{i_{1}} \cdots c_{i_{j}}, \quad n \geq 1 .
$$

Proof. Without loss of generality, we assume that $T=1$. We define a sequence of auxiliary functions $\left\{\varphi_{n}(t)\right\}_{n}$ on $(0,1]$ inductively by

$$
\varphi_{n}(t)=\frac{\varphi_{n-1}(t)-\varphi_{n-1}(0)}{t^{q}}, \quad t \in(0,1] .
$$

We first show that $\varphi_{n}(0):=\lim _{t \rightarrow 0} \varphi_{n}(t)=c_{n}$ by induction.

For $n=1$, it is clear that $\varphi_{0}(t)=\varphi_{0}(0)+\varphi_{1}(t) t^{q}=\varphi_{1}(t) t^{q}$, and by assumption, $\varphi_{1}(t)\left(1-\varphi_{0}(t)\right)^{q}=c_{1}$. This implies $\varphi_{1}(0):=\lim _{t \rightarrow 0} \varphi_{1}(t)=c_{1}$. Assume the statement holds up to $n$. Then by the construction of $\varphi_{k}$ 's, we have

$$
\varphi_{0}(t)=\varphi_{1}(0) t^{q}+\varphi_{2}(0) t^{2 q}+\cdots+\varphi_{n}(0) t^{n q}+\varphi_{n+1}(t) t^{(n+1) q},
$$

also $\varphi_{k}(0)=\lim _{t \rightarrow 0} \varphi_{k}(t)=c_{k}, k \leq n$, and

$$
\lim _{t \rightarrow 0} \varphi_{n+1}(t) t^{q}=\lim _{t \rightarrow 0}\left(\varphi_{n}(t)-\varphi_{n}(0)\right)=0 .
$$

For convenience, we use $C_{q, k}$ to denote the term $\frac{q(q+1) \cdots(q+k)}{(k+1) !}$. Using the binomial series, we have

$$
\left(1-\varphi_{0}(t)\right)^{-q}=1+\sum_{k=0}^{\infty} C_{q, k}\left(\varphi_{0}(t)\right)^{k+1}, \quad t \in[0,1],
$$

We apply this to hypothesis (2.2) and obtain

$$
\varphi_{0}(t)=c_{1} t^{q}\left(1+\sum_{k=0}^{\infty} C_{q, k}\left(\varphi_{0}(t)\right)^{k+1}\right)
$$




$$
\begin{aligned}
= & c_{1} t^{q}\left(1+\sum_{k=0}^{n} C_{q, k}\left(\varphi_{1}(0) t^{q}+\cdots+\varphi_{n}(0) t^{q n}+\varphi_{n+1}(t) t^{q(n+1)}\right)^{k+1}\right) \\
& +\varphi_{0}^{n+1}(t) R_{1, n}(t) \\
= & c_{1} t^{q}\left(1+\sum_{k=0}^{n} C_{q, k}\left(c_{1} t^{q}+\cdots+c_{n} t^{q n}\right)^{k+1}\right)+R_{n}(t)
\end{aligned}
$$

where

$$
R_{n}(t)=\varphi_{n+1}(t) t^{q(n+1)} R_{2, n}(t)+\varphi_{0}^{n+1}(t) R_{1, n}(t),
$$

and $R_{1, n}, R_{2, n}$ are obtained by putting all the excessive terms together. By (2.4), it is direct to check that $R_{n}(t)=o\left(t^{q(n+1)}\right)$ as $t \rightarrow 0$. By regrouping the term in $t^{k q}, 1 \leq k \leq n+1$, we see that the $t^{q(n+1)}$ term has coefficient $c_{n+1}$. Compare it with (2.3), and make use of the induction hypothesis, we have

$$
\varphi_{n+1}(t) t^{q(n+1)}=c_{n+1} t^{q(n+1)}+o\left(t^{q(n+1)}\right), \quad \text { as } t \rightarrow 0 .
$$

Therefore $\varphi_{n+1}(0):=\lim _{t \rightarrow 0} \varphi_{n+1}(t)=c_{n+1}$ exists, and induction follows.

We claim that $\varphi_{n+1}(t)>0$ for all $n \geq 1, t \in(0,1]$. It then follows from $(2.3)$ that

$$
f(t):=\sum_{n=1}^{\infty} c_{n} t^{q n} \leq \varphi_{0}(t)<1, \quad t \in[0,1] .
$$

Suppose on the contrary, there exist $N \geq 1, t_{0} \in(0,1]$ such that $\varphi_{N+1}\left(t_{0}\right) \leq 0$. Since $\varphi_{N+1}(0)=c_{N+1}>0$, and $\varphi_{N+1}(t)$ is continuous in $\left[0, t_{0}\right]$, there exists $t_{1} \in\left[0, t_{0}\right]$ such that $\varphi_{N+1}\left(t_{1}\right)=0$. By $(2.3)$, we have

$$
\varphi_{0}\left(t_{1}\right)=c_{1} t_{1}^{q}+c_{2} t_{1}^{2 q}+\cdots+c_{N} t_{1}^{q N} .
$$

Noting that $R_{1, N}\left(t_{1}\right)>0$ and $\varphi_{N+1}\left(t_{1}\right)=0$, we obtain $R_{N}\left(t_{1}\right)>0$. It follows from (2.5) that $\varphi_{0}\left(t_{1}\right)>c_{1} t_{1}^{q}+c_{2} t_{1}^{2 q}+\cdots+c_{N} t_{1}^{q N}$. This contradicts with (2.7). Hence our claim holds.

It remains to show that $\varphi_{0}(t)=\sum_{n=0}^{\infty} c_{n} t^{q n}$. To this end, we consider a new function

$$
F(t, x):=F_{t}(x)=x(1-x)^{q}-c_{1} t^{q}, \quad x \in[0,1] .
$$

Since $F_{t}^{\prime}(x)=(1-x)^{q-1}(1-(q+1) x), F_{t}(x)$ is strictly increasing in $\left[0, \frac{1}{q+1}\right]$ (and strictly decreasing in $\left.\left[\frac{1}{q+1}, 1\right]\right)$. By the implicit function theorem, there is a unique $0 \leq x(t) \leq \frac{1}{q+1}, 0 \leq t \leq 1$ such that $F(t, x(t))=0$ with $x(0)=0$. Now observe that $\varphi_{0}(0)=0, F\left(t, \varphi_{0}(t)\right)=0$ by (2.2). Also for $f(t)=\sum_{n=0}^{\infty} c_{n} t^{q n}$, we have $f(0)=0$ and $F(t, f(t))=0$, as it follows from (2.6) that

$$
\begin{aligned}
c_{1} t^{q}(1-f(t))^{-q} & =c_{1} t^{q}\left(1+\sum_{k=0}^{\infty} C_{q, k}(f(t))^{k+1}\right) \\
& =c_{1} t^{q}\left(1+\sum_{k=0}^{\infty} C_{q, k}\left(c_{1} t^{q}+c_{2} t^{2 q}+c_{3} t^{3 q}+\cdots\right)^{k+1}\right) \\
& =c_{1} t^{q}\left(1+C_{q, 0} c_{1} t^{q}+\left(C_{q, 0} c_{2}+C_{q, 1} c_{1}^{2}\right) t^{2 q}+\cdots\right) \\
& =c_{1} t^{q}+c_{2} t^{2 q}+c_{3} t^{3 q}+\cdots=f(t) .
\end{aligned}
$$

The uniqueness implies that $\varphi_{0}(t)=f(t)$. 


\section{Proof of Theorem 1.1}

For simplicity, we will use the following notations: $f(\epsilon) \lesssim g(\epsilon)$ means $f(\epsilon) \leq$ $C g(\epsilon)$ for some constant $C>0 ; f(\epsilon) \asymp g(\epsilon)$ means $f(\epsilon) \lesssim g(\epsilon)$ and $g(\epsilon) \lesssim f(\epsilon)$. We give a more complete version of Theorem 1.1.

Theorem 3.1. The slit $\Gamma^{p}$ is generated by the Loewner equation with driving function $\lambda(t)$ given by

$$
\lambda(t)=c^{p}\left(\sum_{n=1}^{\infty} c_{n} t^{q n}\right)^{p}\left(1-2 \sum_{n=1}^{\infty} c_{n} t^{q n}\right), \quad t \in[0, T]
$$

for some $T>0$, where $c=\frac{4(p+1) \pi}{p(2 p+1)}, q=\frac{1}{2 p+1}$, and

$$
c_{n+1}=c_{1} \sum_{j=1}^{n} \sum_{\substack{i_{1}+\cdots+i_{j}=n \\ i_{1}, \cdots, i_{j} \geq 1}} \frac{q(q+1) \cdots(q+j-1)}{j !} c_{i_{1}} \cdots c_{i_{j}}, \quad n \geq 1
$$

with $c_{1}=c^{-1}\left(\frac{4(2 p+1) \pi}{p^{2}}\right)^{q}$. In particular, we have

$$
\lambda(t)=\left(c c_{1}\right)^{p} t^{\frac{p}{2 p+1}}+o\left(t^{\frac{p}{2 p+1}}\right), \quad \text { as } t \rightarrow 0 .
$$

We will divide the proof of the theorem into two lemmas to obtain the functional equation in Lemma 2.4.

Let $\gamma(t): 0 \leq t \leq T$ be the parameterization of $\Gamma^{p}$ such that hcap $\gamma(0, t]=2 t$. Let $g_{t}$ be the solution of the Loewner equation which maps $\mathbf{H} \backslash \gamma(0, t]$ onto $\mathbf{H}$, and let $f_{t}$ be the inverse of $g_{t}$. Let $\lambda(t)=g_{t}(\gamma(t))$. Since $g_{t}$ is well-defined in $\mathbf{R} \backslash\{0\}$, the two functions $\alpha(t)=g_{t}(0-)$ and $\beta(t)=g_{t}(0+)$ are also well defined. When there is no confusion, we suppress the variable $t$ and just write $\lambda, \alpha$ and $\beta$ for brevity.

First, we will give an integral expression of $f_{t}$ as the following.

Lemma 3.2. For $p>0$, let $w(z)=z^{-\frac{1}{p}}$ (with the branch satisfying $\ln 1=0$ ), and let $h_{t}=w \circ f_{t}$ be defined on $\mathbf{H}$. Then

$$
h_{t}(z)-h_{t}\left(z_{0}\right)=-\frac{1}{p} \int_{z_{0}}^{z}(\xi-\alpha)^{-\frac{1}{p}-1}(\xi-\lambda)(\xi-\beta)^{-1} d \xi .
$$

for any fixed $z_{0} \in \mathbf{H}$.

Proof. We write $w(z)=\psi \circ \phi(z), z \in \mathbf{H}$, where $\psi(z)=1 / z, \phi(z)=z^{\frac{1}{p}}$. Noting that $\Gamma$ is a circular arc, let $\Gamma^{\prime}$ be the subarc of $\Gamma$ on $\left\{-\frac{\pi}{2} \leq \theta \leq \frac{\pi}{2}\left(\theta_{p}-1\right)\right\}$ (where $\theta_{p}$ is defined in the Introduction). Then we have

$$
w\left(\Gamma^{p}\right)=\psi \circ \phi\left(\Gamma^{p}\right)=\psi\left(\Gamma^{\prime}\right)=\{x-i / 2: x \geq \operatorname{Re} w(\gamma(T))\} .
$$

Clearly $w(\mathbf{H})=\left\{r e^{i \theta}: r>0,-\frac{\pi}{p}<\theta<0\right\}$. It follows that for $p \geq 1 / 2, w$ maps $\mathbf{H} \backslash \gamma(0, t]$ conformally onto the domain

$$
M_{t}=\left\{r e^{i \theta}: r>0,-\frac{\pi}{p}<\theta<0\right\} \backslash\{x-i / 2: x \geq \operatorname{Re} w(\gamma(t))\} ;
$$

but for $0 \leq p<1 / 2$, $w$ is multivalued (as $w(\mathbf{H})$ wraps around). We will divide our proof into two cases.

Case 1: $p \geq 1 / 2$. Define $h_{t}:=w \circ f_{t}=\left(f_{t}\right)^{-\frac{1}{p}}$, then it maps $\mathbf{H}$ conformally onto $M_{t}$. By applying the Christoffel-Schwarz formula to any fixed $z_{0} \in \mathbf{H}$, we can 
express $h_{t}$ as

$$
h_{t}(z)-h_{t}\left(z_{0}\right)=b \int_{z_{0}}^{z}(\xi-\alpha)^{-\frac{1}{p}-1}(\xi-\lambda)(\xi-\beta)^{-1} d \xi .
$$

To determine $b$, we observe that

$$
h_{t}^{\prime}(z)=b(z-\alpha)^{-\frac{1}{p}-1}(z-\lambda)(z-\beta)^{-1} .
$$

As $h_{t}^{\prime}(z)=\left(w \circ f_{t}\right)^{\prime}=\left(-\frac{1}{p}\right)\left[f_{t}(z)\right]^{-\frac{1}{p}-1} f_{t}^{\prime}(z)$, we have

$$
f_{t}^{\prime}(z)=(-b p)\left[f_{t}(z)\right]^{\frac{1}{p}+1}(z-\alpha)^{-\frac{1}{p}-1}(z-\lambda)(z-\beta)^{-1} .
$$

Noting that

$$
f_{t}(z)=z-\frac{2 t}{z}+o\left(\frac{1}{z}\right), \quad \text { as } z \rightarrow \infty,
$$

we have $f_{t}^{\prime}(z) \rightarrow 1$ and $f_{t}(z) / z \rightarrow 1$ as $z \rightarrow \infty$. It follows that $-b p=1$, i.e., $b=-\frac{1}{p}$.

Case 2: $0<p<1 / 2$. We need to adjust $M_{t}$ as a polygon in some Riemann surface to apply the Christoffel-Schwarz formula. Let $S:=\mathbf{R}^{+} \times \mathbf{R}$ be the Riemann surface in the following sense:

(i) $S=\bigcup_{m, n \in \mathbf{Z}}\left(U_{m} \cup V_{n}\right)$, where $U_{m}=\mathbf{R}^{+} \times(2 m \pi, 2(m+1) \pi), V_{n}=\mathbf{R}^{+} \times$ $((2 n+1) \pi,(2 n+3) \pi)$. For each $m, n \in \mathbf{Z}$, define

$$
\phi_{m}: U_{m} \rightarrow \mathbf{C},(r, \theta) \mapsto r e^{i \theta}, \quad \psi_{n}: V_{n} \rightarrow \mathbf{C},(r, \theta) \mapsto r e^{i \theta} .
$$

(ii) If $U_{m}$ and $V_{n}$ intersect for some $m, n \in \mathbf{Z}$, then the transition map

$$
\Phi_{m, n}=\phi_{m} \circ \psi_{n}^{-1}: \psi_{n}\left(U_{m} \cap V_{n}\right) \rightarrow \phi_{m}\left(U_{m} \cap V_{n}\right)
$$

is a conformal map from $U_{m} \cap V_{n}$ onto itself.

Define the map $w^{*}: \mathbf{H} \rightarrow S, w^{*}\left(r e^{i \theta}\right)=\left(r^{-\frac{1}{p}},-\frac{\theta}{p}\right)$ for $r>0, \theta \in(0, \pi)$. Following the notations in Case 1 , we still denote by $M_{t}$ the Riemann surface $w^{*}(\mathbf{H} \backslash \gamma[0, t])$, and denote $w^{*}$ by $w$. Obviously, $w$ is $1-1$ from $\mathbf{H} \backslash \gamma[0, t]$ onto $M_{t}$, and the boundary of $M_{t}$ consists of three rays. It follows from [3] and [4] that the Christoffel-Schwarz formula (3.1) still holds for this case, and the same proof can be carried through.

Lemma 3.3. With the above notations, we have $\alpha(t)<0$, and the following identities for $\lambda(t), \alpha(t)$ and $\beta(t)$ :

$$
\lambda=\left(\frac{1}{p}+1\right) \alpha+\beta, \quad \beta=\alpha+C^{\prime}(-\alpha)^{\frac{p}{p+1}},
$$

where $C^{\prime}=\left(\frac{2(p+1) \pi}{p^{2}}\right)^{\frac{p}{p+1}}$, and

$$
(-\alpha)^{\frac{2 p+1}{p+1}}\left(b-(-\alpha)^{\frac{1}{p+1}}\right)=\delta t,
$$

where $b=\frac{2 p}{2 p+1} C^{\prime}, \delta=\frac{4 p}{p+1}$.

Proof. Noting that $h_{t}(\infty)=0$, by letting $z_{0} \rightarrow \infty$, and making a change of variable $u=\xi^{-1}$, we have

$$
h_{t}(z)=\int_{0}^{\frac{1}{z}} F(u) d u \quad \text { where } \quad F(u)=\frac{(1-\lambda u)}{p(1-\alpha u)^{1+\frac{1}{p}}(1-\beta u) u^{1-\frac{1}{p}}} .
$$


We expand the first three terms of $F(u)$ and obtain

$$
F(u)=\frac{1}{p} u^{\frac{1}{p}-1}\left(1+a_{1} u+a_{2} u^{2}+o\left(u^{2}\right)\right) \quad \text { as } \quad u \rightarrow 0,
$$

where $a_{1}=\left(1+\frac{1}{p}\right) \alpha+\beta-\lambda$ and $a_{2}=\left(1+\frac{1}{p}\right) \alpha \beta-\left(1+\frac{1}{p}\right) \alpha \lambda-\beta \lambda+\beta^{2}+\left(1+\frac{1}{p}\right)\left(1+\frac{1}{2 p}\right) \alpha^{2}$. Integrating $F(u)$ and noting that $f_{t}(z)=\left(h_{t}(z)\right)^{-p}$, we conclude that

$$
f_{t}(z)=z-\frac{a_{1} p}{p+1}-\left(\frac{a_{2} p}{2 p+1}-\frac{a_{1}^{2} p}{2 p+2}\right) \frac{1}{z}+o\left(\frac{1}{z}\right) \quad \text { as } \quad z \rightarrow \infty .
$$

Hence it follows from (3.2) that $a_{1}=0, a_{2}=\frac{(4 p+2) t}{p}$. By equating the two expressions on $a_{1}$, we have

$$
\beta-\lambda=-\left(\frac{1}{p}+1\right) \alpha
$$

This implies $\alpha<0$, and also the first identity in (3.3) follows. Similarly by equating the two expressions of $a_{2}$, and use (3.5) to substitute away the $\lambda$, we obtain

$$
\alpha\left(\beta+\frac{\alpha}{2 p}\right)=-\frac{(4 p+2) t}{p+1} .
$$

To prove the second identity, we use partial fractions to express $h_{t}(z)$ as

$$
h_{t}(z)-h_{t}\left(z_{0}\right)=\frac{\alpha-\lambda}{p(\beta-\alpha)} \int_{z_{0}-\alpha}^{z-\alpha} \xi^{-\frac{1}{p}-1} d \xi+\frac{\beta-\lambda}{p(\beta-\alpha)} \int_{z_{0}-\alpha}^{z-\alpha} \frac{\xi^{-\frac{1}{p}} d \xi}{(\beta-\alpha)-\xi} .
$$

Let $z_{0}=2 \beta-\lambda, z=\lambda$, then the first part of the integral is real. By observing that $h_{t}$ maps $\mathbf{R}$ onto the boundary of $M_{t}$, we see that $h_{t}(2 \beta-\lambda)$ is real and $\operatorname{Im} h_{t}(\lambda)=-\frac{1}{2}$. This yields

$$
\frac{1}{2}=\frac{\beta-\lambda}{p(\beta-\alpha)^{1+\frac{1}{p}}} \cdot \operatorname{Im} \int_{1-s}^{1+s} \frac{d \xi}{\xi^{\frac{1}{p}}(1-\xi)},
$$

where $s=\frac{\beta-\lambda}{\beta-\alpha}$. We claim that the last expression equals $\pi$. Then the above identity together with (3.5) imply the second identity in (3.3).

To prove the claim, we choose the integral path $\Lambda: \xi=1+s e^{i \theta}, 0 \leq \theta \leq \pi$, the imaginary part of the above integral is equal to

$$
\operatorname{Re} \int_{0}^{\pi} \frac{d \theta}{\left(1+s e^{i \theta}\right)^{\frac{1}{p}}}=\pi
$$

The value $\pi$ is a simple consequence of the power series expansion of $(1+z)^{-\frac{1}{p}}$ at $z=0$, noting that $0<s<1$.

Putting the second identity in (3.3) into (3.6), we have the last identity of the lemma.

Remark 3.4. The formulae (3.3) and (3.4) for $p=1$ are given in [16].

Proof of Theorem 3.1. By (3.3),

$$
\lambda=\left(\frac{1}{p}+2\right) \alpha+C^{\prime}(-\alpha)^{\frac{p}{p+1}}
$$

Therefore it suffices to find the appropriate expression of $(-\alpha)$ in terms of $t$ on $[0$, T]. In view of (2.2) and (3.4), we let

$$
\varphi_{0}(t)=\frac{1}{b}(-\alpha)^{\frac{1}{p+1}}, \quad t \in[0, T] .
$$


It follows from Theorem 2.2 that $\beta-\alpha \asymp \operatorname{diam} \gamma[0, t]$. By (3.3), $-\alpha \asymp \beta-\lambda \leq \beta-\alpha$ holds. Thus we have $\lim _{t \rightarrow 0} \varphi_{0}(t)=0$. Putting (3.8) into (3.4) yields $b^{2 p+2} \varphi_{0}^{2 p+1}(t)(1-$ $\left.\varphi_{0}(t)\right)=\delta t$. By letting $q=\frac{1}{2 p+1}$ and simplify, we arrive

$$
\varphi_{0}(t)\left(1-\varphi_{0}(t)\right)^{q}=c_{1} t^{q}, \quad t \in[0, T],
$$

where $c_{1}=\left(\frac{\delta}{b}\right)^{q} b^{-1}$ has the expression in Theorem 3.1. Equation (3.9) fulfils the conditions in Lemma 2.4, and hence $\varphi_{0}(t)=\sum_{n=1}^{\infty} c_{n} t^{q n}$ as stated. In view of (3.7) and (3.8), we have

$$
\lambda=c^{p}\left(\sum_{n=1}^{\infty} c_{n} t^{q n}\right)^{p}\left(1-2 \sum_{n=1}^{\infty} c_{n} t^{q n}\right), \quad t \in[0, T] .
$$

This proves the theorem.

\section{Proof of Theorem 1.2}

To prove Theorem 1.2, we need the following lemma:

Lemma 4.1. Let $r>1, c>0$, and $\gamma_{c}(x)=x+i c x^{r}$. Then hcap $\left(\gamma_{c}(0, x]\right) \asymp$ $x^{r+1}, x \in(0,1 / 2]$.

Proof. Let $\ell_{c}(x)=\operatorname{hcap}\left(\gamma_{c}(0, x]\right)$. Noting that

$$
c^{\frac{1}{r-1}} \gamma_{c}(0, x]=\gamma_{1}\left(0, c^{\frac{1}{r-1}} x\right]
$$

and using (2.1), we have $\ell_{1}\left(c^{\frac{1}{r-1}} x\right)=c^{\frac{2}{r-1}} \ell_{c}(x)$. Therefore it suffices to show that $\ell_{1}(x) \asymp x^{r+1}$. It follows from Theorem 2.1 that $\ell_{1}(x) \asymp \operatorname{hsiz}\left(\gamma_{1}(0, x]\right)$. Hence it suffices to show that

$$
\operatorname{hsiz}\left(\gamma_{1}(0, x]\right) \asymp x^{r+1} .
$$

Let

$$
D_{c}(x)=\left\{u+i v: 0 \leq u \leq x, 0 \leq v \leq c u^{r}\right\} \cup B\left(x+i c x^{r}, c x^{r}\right) .
$$

We claim that there exists a constant $C>1$ such that $\operatorname{dist}\left(x+i x^{r}, \gamma_{C}\right) \geq x^{r}$ holds for each $x \in(0,1 / 2]$. This implies that for $x \in(0,1 / 2]$,

$$
D_{1}(x) \subset \bigcup_{0 \leq u \leq x} B\left(u+i u^{r}, u^{r}\right) \subset D_{C}(x) .
$$

Hence $\operatorname{area}\left(D_{1}(x)\right) \leq \operatorname{hsiz}\left(\gamma_{1}(0, x]\right) \leq \operatorname{area}\left(D_{C}(x)\right)$. Since area $\left(D_{1}(x)\right) \asymp x^{r+1} \asymp$ area $\left(D_{C}(x)\right)$ (by direct calculation), the estimation (4.1) holds.

Now, we prove our claim. For each fixed $x \in(0,1 / 2]$, let $c(x)$ be defined so that the curve $\gamma_{c(x)}(u)=u+i c(x) u^{r}$ tangents to the ball $B\left(x+i x^{r}, x^{r}\right)$ at $z_{x}=\bar{x}+i c(x) \bar{x}^{r}$. Then $\operatorname{dist}\left(x+i x^{r}, z_{x}\right)=x^{r}$, and $\left(c(x) \bar{x}^{r}-x^{r}\right)^{2}+(\bar{x}-x)^{2}=x^{2 r}$. It follows that

$$
c^{2}(x) \bar{x}^{2 r} \leq 2 c(x)(x \bar{x})^{r} .
$$

As $x-x^{r} \leq \bar{x} \leq x, c(x) \leq 2\left(1-2^{1-r}\right)^{-r}:=C$ for $x \in(0,1 / 2]$, and $\operatorname{dist}\left(x+i x^{r}, \gamma_{C}\right) \geq$ $x^{r}$ holds for each $x \in(0,1 / 2]$.

Proof of Theorem 1.2. Let $\left\{K_{t}\right\}_{t \in[0, T]}$ be the growing hulls generated by the Loewner equation with driving function $\lambda(t)$, and let $K_{T} \subset D_{r}(a, b)=\{x+i y: x>$ $\left.0, a x^{r}<y<b x^{r}\right\}$. Let

$$
x(t)=\sup \left\{x: x+i y \in K_{t}\right\}, \quad t \in[0, T] .
$$


We claim that $x(t) \asymp t^{\frac{1}{r+1}}$. As $K_{t} \subset D_{r}(a, b)$, by the definition of hsiz $(K)$, we have

$$
\operatorname{hsiz}\left(\gamma_{a}(0, x(t)]\right) \leq \operatorname{hsiz}\left(K_{t}\right) \leq \operatorname{hsiz}\left(\gamma_{b}(0, x(t)]\right) .
$$

It follows from Theorem 2.1 that

$$
\ell_{a}(x(t)) \lesssim \operatorname{hcap}\left(K_{t}\right)=2 t \lesssim \ell_{b}(x(t)) .
$$

By Lemma 4.1, $x(t) \asymp t^{\frac{1}{r+1}}$ holds.

Now let $A_{t}=\left\{u+i v: 0 \leq u \leq x(t), 0 \leq v \leq b u^{r}\right\}$. Since $K_{t} \subset D_{r}(a, b)$, we have $x(t) \leq \operatorname{diam} K_{t} \leq \operatorname{diam} A_{t} \asymp x(t)$, which implies that

$$
x(t) \asymp \operatorname{diam} K_{t} .
$$

Let $I_{t}=\left[g_{t}\left(\sigma_{t}-\right), g_{t}\left(\tau_{t}+\right)\right]$ with $\sigma_{t}=\inf \left(\bar{K}_{t} \cap \mathbf{R}\right), \tau_{t}=\sup \left(\bar{K}_{t} \cap \mathbf{R}\right)$. Theorem 2.2 implies that $\operatorname{diam} K_{t} \asymp \operatorname{diam} I_{t}$. This and (4.2) imply $x(t) \asymp \operatorname{diam} I_{t}$. Noting that $\lambda(t) \in I_{t}$ and $\lambda(0)=0 \in I_{t}$, we have $|\lambda(t)| \lesssim x(t) \asymp t^{\frac{1}{r+1}}$. This implies $\lim \sup _{t \rightarrow 0}|\lambda(t)| / t^{\frac{1}{r+1}}<\infty$.

\section{References}

[1] De Branges, L.: A proof of the Bieberbach conjecture. - Acta Math. 154, 1985, 137-152.

[2] Earle, C., and A. Epstein: Quasiconformal variation of slit domains. - Proc. Amer. Math. Soc. 129, 2001, 3363-3372.

[3] Gilbarg, D.: A generalization of the Schwarz-Christoffel transformation. - Proc. Nat. Acad. Sci. 35, 1949, 609-612.

[4] Goodman, A.: On the Schwarz-Christoffel transformation and $p$-valent functions. - Trans. Amer. Math. Soc. 68, 1950, 204-223.

[5] Ivanov, G., D. Prokhorov, and A. VasiL'Ev: Non-slit and singular solutions to the Löwner equation. - Bull. Sci. Math. 136, 2012, 328-341.

[6] Kager, W., B. Nienhuis, and L. Kadanoff: Exact solutions for Loewner evolutions. - J. Statist. Phys. 115, 2004, 805-822.

[7] Lalley, S., G. Lawler, and H. Narayanan: Geometric interpretation of half-plane capacity. - Electron. Commun. Prob. 14, 2009, 566-571.

[8] LawleR, G.: Conformally invariant processes in the plane. - Amer. Math. Soc., 2005.

[9] Lawler, G., O. Schramm, and W. Werner: Values of Brownian intersection exponents I: half-plane exponents. - Acta Math. 187, 2001, 237-273.

[10] Lawler, G., O. Schramm, and W. Werner: Values of Brownian intersection exponents II: plane exponents. - Acta Math. 187, 2001, 275-308.

[11] Lawler, G., O. Schramm, W. Werner: Values of Brownian intersection exponents III: two-sided exponents. - Ann. Inst. H. Poincaré Probab. Statist. 38, 2002, 109-123.

[12] Lind, J.: A sharp condition for the Loewner equation to generate slits, - Ann. Acad. Sci. Fenn. Math. 30, 2005, 143-158.

[13] Lind, J., D. Marshall, and S. Rohde: Collisions and spirals of Loewner traces. - Duke Math. 154, 2010, 527-573.

[14] Lind, J., and H. Tran: Regularity of Loewner curves. - Eprint arXiv, 2014.

[15] Marshall, D., and S. Rohde: The Loewner differential equation and slit mappings. - J. Amer. Math. Soc. 18, 2005, 763-778.

[16] Prokhorov, D., and A. VAsiL'Ev: Singular and tangent slit solutions to the Löwner equation. - In: Analysis and Mathematical Physics, Trends Math., Birkhäuser, 2009, 455-463. 
[17] Rohde, S., and C. Wong: Half-plane capacity and conformal radius. - Proc. Amer. Math. Soc. 142, 2014, 931-938.

[18] Schleissinger, S.: The multiple-slit version of Loewner's differential equation and pointwise Hölder continuity of driving functions. - Ann. Acad. Sci. Fenn. Math. 37, 2012, 191-201.

[19] Schramm, O.: Scaling limits of loop-erased random walks and uniform spanning trees. - Israel J. Math. 118, 2000, 221-288.

[20] Smirnov, S.: Critical percolation in the plane: conformal invariance, Cardy's formula, scaling limits. - C. R. Acad. Sci. Paris 333, 2001, 239-244.

[21] Smirnov, S.: Critical percolation and conformal invariance. - In: XIVth International Congress on Mathematical Physics, 2003, 99-112.

[22] Smirnov, S.: Towards conformal invariance of 2D lattice models. - In: Proceedings of the International Congress of Mathematicians 2, 2006, 1421-1451.

[23] Smirnov, S., and W. Werner: Critical exponents for two-dimensional percolation. - Math. Res. Lett. 8, 2001, 729-744.

[24] Wong, C.: Smoothess of Loewner slits. - Trans. Amer. Math. Soc. 366, 2014, 1475-1496.

[25] Wu, H. H., and X. H. Dong: Driving functions and traces of the Loewner equation. - Sci. China Math. 57, 2014, 1615-1624.

Received 5 September $2015 \bullet$ Accepted 18 January 2016 